\title{
Characterization of bovine glucose transporter 1 kinetics and substrate specificities in Xenopus oocytes
}

\author{
P. A. Bentley, ${ }^{* 1}$ Y. Shao, ${ }^{*}$ Y. Misra, ${ }^{*}$ A. D. Morielli, $\dagger$ and F.-Q. Zhao ${ }^{\star 2}$ \\ *Laboratory of Lactation Physiology, Department of Animal Science, and \\ †Department of Pharmacology, College of Medicine, University of Vermont, Burlington 05405
}

\begin{abstract}
Glucose is an essential substrate for lactose synthesis and an important energy source in milk production. Glucose uptake in the mammary gland, therefore, plays a critical role in milk synthesis. Facilitative glucose transporters (GLUT) mediate glucose uptake in the mammary gland. Glucose transporter 1 (GLUT1) is the major facilitative glucose transporter expressed in the bovine mammary gland and has been shown to localize to the basolateral membrane of mammary epithelial cells. Glucose transporter 1 is, therefore, thought to play a major role in glucose uptake during lactation. The objective of this study was to determine the transport kinetic properties and substrate specificity of bovine GLUT1 using the Xenopus oocyte model. Bovine GLUT1 (bGLUT1) was expressed in Xenopus oocytes by microinjection of in vitro transcribed cRNA and was found to be localized to the plasma membrane, which resulted in increased glucose uptake. This bGLUT1mediated glucose uptake was dramatically inhibited by specific facilitative glucose transport inhibitors, cytochalasin B, and phloretin. Kinetic analysis of bovine and human GLUT1 was conducted under zero-trans conditions using radio-labeled 2-deoxy-D-glucose and the principles of Michaelis-Menten kinetics. Bovine GLUT1 exhibited a Michaelis constant $\left(K_{m}\right)$ of $9.8 \pm$ $3.0 \mathrm{~m} M$ for 2-deoxy-D-glucose, similar to $11.7 \pm 3.7$ $\mathrm{m} M$ for human GLUT1. Transport by bGLUT1 was inhibited by mannose and galactose, but not fructose, indicating that bGLUT1 may also be able to transport mannose and galactose. Our data provides functional insight into the transport properties of bGLUT1 in taking up glucose across mammary epithelial cells for milk synthesis.
\end{abstract}

Key words: bovine glucose transporter, glucose transport, kinetics, substrate specificity

\footnotetext{
Received April 8, 2011.

Accepted November 3, 2011.

${ }^{1}$ Current address: Department of Agricultural, Food and Nutritional Science, University of Alberta, 410 Agriculture/Forestry Centre, Edmonton, AB T6G 2P5, Canada.

${ }^{2}$ Corresponding author: Feng-Qi.Zhao@uvm.edu
}

\section{INTRODUCTION}

Glucose is the primary precursor of lactose, which is a principle component of milk and functions as the primary osmotic regulator of milk volume (Peaker, 1977). The mammary gland uses approximately $3 \mathrm{~kg}$ of glucose for every $40 \mathrm{~kg}$ of milk produced. Mammary epithelial cells lack the ability to synthesize glucose; therefore, glucose must be obtained from the blood. Glucose transport into the mammary epithelial cells is primarily mediated by the energy-independent facilitative glucose transporters (GLUT; gene name: SLC2A; Zhao and Keating, 2007a,b). The GLUT family currently includes 13 members and its structure is characterized by 12 transmembrane domains and a single N-glycosylation site (Mueckler et al., 1985). Glucose transporter 1 (GLUT1) has been shown to be the major GLUT isoform expressed in the bovine mammary gland and its expression is developmentally regulated (Zhao et al., 1993; Zhao and Keating, 2007a). From $40 \mathrm{~d}$ before parturition to $7 \mathrm{~d}$ postcalving, GLUT1 expression in the bovine mammary gland increases more than 100-fold (Zhao and Keating, 2007a; Finucane et al., 2008). This dramatic increase in GLUT1 expression may be responsible for supplying the mammary gland with increased glucose needed during lactation.

Individual GLUT vary in tissue distribution, kinetic characteristics (Michaelis constant, $\boldsymbol{K}_{m}$, the substrate concentration at which the reaction rate is half of the maximum rate, $\boldsymbol{V}_{\max }$, and substrate specificities, implying that each transporter plays a distinct role in tissue glucose utilization and maintenance of body glucose homeostasis. For instance, whereas the human and rodent GLUT1 have a $K_{m}$ of 6.9 to $17 \mathrm{~m} M$ for D-glucose (Gould et al., 1991; Burant and Bell, 1992; Nishimura et al., 1993), hepatocyte GLUT (GLUT2) has a 2 to 10fold higher $K_{m}$ and a higher $V_{\max }$ to allow glucose efflux following gluconeogenesis (Gould et al., 1991; Burant and Bell, 1992; Colville et al., 1993). Glucose transporter 3 and GLUT4, with lower $K_{m}$ values, mediate the uptake of glucose by the brain and the insulin-regulated glucose uptake by skeletal muscle, respectively (Burant and Bell, 1992; Colville et al., 1993; Nishimura 
et al., 1993). Glucose transporter 5 has a high affinity for fructose, with a poor ability to transport glucose (Corpe et al., 2002). In addition, the transport kinetics and substrate specificity of some transporters have been shown to differ between species. For instance, although the pig sodium/glucose cotransporter 3 (SGLT3) shows a tightly coupled sodium and glucose transport activity, its human homolog, hSGLT3, does not have this characteristic (Wright and Turk, 2004).

Because most mammalian cells express several different GLUT with high basal glucose transport activity, it is difficult to determine the transport kinetics and the substrate specificity of individual GLUT in situ. Characterization of the transport kinetics of individual transporters has been widely carried out in Xenopus oocytes by overexpressing the transporter (Keller et al., 1989; Keller and Mueckler, 1990; Colville et al., 1993; Nishimura et al., 1993). Xenopus oocytes exhibit extremely low levels of basal glucose transport activity and, thus, offer an ideal system for expression and functional characterization of heterologous GLUT using radiolabeled nonmetabolizable glucose analogs such as 2-deoxy-D-glucose (2-DG) and 3-O-methylglucose. The general transport properties of GLUT derived from expression studies in Xenopus oocytes do relate well to the known characteristics of glucose transport in various mammalian tissues.

Considering that the normal blood glucose levels in bovine (2.5-3.5 $\mathrm{mM})$, being a ruminant, are generally lower than that of human (3.6-5.8 $\mathrm{mM})$ and other nonruminant animals, we hypothesize that the bovine GLUT1 (bGLUT1), the isoform ubiquitously distributed and responsible for basal glucose uptake of most tissues, has a lower $K_{m}$ than does the human GLUT1 (hGLUT1). The objectives of this study were to characterize the transport kinetics of bGLUT1 and to compare the kinetics properties of GLUT1 between bovines and humans. We further aimed to determine bGLUT1 substrate specificities. Knowing the characteristics of bGLUT1, the predominant isoform in the lactating bovine mammary gland, will provide insights into the physiological function of GLUT1 in bovine mammary cells and its roles in supporting milk synthesis.

\section{MATERIALS AND METHODS}

\section{Plasmid Constructs}

The hGLUT1 plasmid construct, pSP64T-hGLUT1, was kindly donated by Gwyn Gould (Gould et al., 1991). The bGLUT1 cDNA (Zhao et al., 2004) was cloned into the Xenopus expression vector SP64T as follows: the pSP64T-hGLUT1 was first digested with BglII (New England Biolabs Inc., Ipswich, MA) over- night at $37^{\circ} \mathrm{C}$ to remove the hGLUT1 insert and the vector product was then purified by phenol extraction and ethanol precipitation, resolved on a $1 \%$ agarose gel, and isolated using a QIAquick Gel Extraction Kit (Qiagen Inc., Valencia, CA). Bovine GLUT1 cDNA was excised from the pcDNA3.1(-)-bGLUT1 plasmid by digestion with $\mathrm{XbaI}$ and HindIII (New England Biolabs Inc.) and purified by gel extraction. Bovine GLUT1 was then digested with BamHI and ligated into the pSP64T vector at the BglII site between the $89 \mathrm{bp}$ $5^{\prime}$ - and 141 bp $3^{\prime}$-Xenopus $\beta$-globin flanking sequences. The resulting pSP64T-bGLUT1 construct was verified by restriction digestion and sequencing.

\section{In Vitro Transcription of Human and Bovine GLUT1-pSP64T Constructs}

The hGLUT1 and bGLUT1-pSP64T constructs were in vitro transcribed and $5^{\prime}$ capped using an SP6 RNA polymerase and the mMessage mMachine Kit (Ambion Inc., Austin, TX). The plasmid DNA was first linearized by restriction digestion with SalI (New England Biolabs Inc.). Linearized plasmid DNA $(1 \mu \mathrm{g})$ was transcribed as per manufacturer's instructions, which included DNase treatment for $15 \mathrm{~min}$. The resulting cRNA was recovered using MEGAclear Kit (Ambion Inc.) and was eluted in $50 \mu \mathrm{L}$ of DNase- and RNase-free water. Complementary RNA concentration was measured by optical density $(260 / 280 \mathrm{~nm})$ using the NanoDrop ND-1000 (Thermo Fisher Scientific, Wilmington, DE) and diluted to $300 \mathrm{ng} / \mathrm{uL}$. Complementary RNA quality was assessed by the Agilent 2100 Bioanalyzer (Agilent Technologies Inc., Santa Clara, CA) under reducing conditions.

\section{Xenopus Oocyte Harvest}

The use of Xenopus and the experimental procedures were approved by the University of Vermont Institutional Animal Care and Use Committee (Burlington). Female Xenopus laevis were maintained at the Small Animal Care Facility of the University of Vermont on a 12-h light/dark cycle and fed a standard diet of bovine liver pellets. Animals were anesthetized for oocyte removal by submersion in $1 \mathrm{~g} / \mathrm{L}$ of tricaine methanesulfonate (Sigma, St. Louis, MO; pH 7.0) in aged and dechlorinated water for $15 \mathrm{~min}$ or until motor reflex was undetectable. Incisions were made parasagittally on the abdomen using a \#11 scalpel blade. Oocytes were removed, severed by scissors and placed in calcium-free OR2 media $\left(82.5 \mathrm{~m} M \mathrm{NaCl}, 2 \mathrm{~m} M \mathrm{KCl}, 1 \mathrm{~m} M \mathrm{MgCl}_{2}\right.$, and $5 \mathrm{~m} M$ HEPES, $\mathrm{pH} 7.5$ ).

Excised oocytes were washed 3 times in $40 \mathrm{~mL}$ of calcium-free OR2 and then incubated in $1 \mathrm{mg} / \mathrm{mL}$ of 
Collagenase Type II (Sigma) in calcium-free OR2 for 1 $\mathrm{h}$ at $22^{\circ} \mathrm{C}$ with agitation. Oocytes were again washed 3 times in $40 \mathrm{~mL}$ of calcium-free OR2 and then transferred to a Petri dish and maintained at $18^{\circ} \mathrm{C}$ in Barth's media [ $88 \mathrm{~m} M \mathrm{NaCl}, 1 \mathrm{~m} M \mathrm{KCl}, 2.4 \mathrm{~m} M \mathrm{NaHCO}_{3}, 0.82$ $\mathrm{m} M \mathrm{MgSO}_{4}, 0.41 \mathrm{~m} M \mathrm{CaCl}_{2}, 0.33 \mathrm{mM} \mathrm{Ca}\left(\mathrm{NO}_{3}\right)_{2}$, and $5 \mathrm{~m} M$ HEPES, pH 7.6] with $10 \mu \mathrm{g} / \mathrm{mL}$ of penicillin and $10 \mathrm{IU} / \mathrm{mL}$ of streptomycin (Invitrogen Corp., Carlsbad, $\mathrm{CA}$ ). Oocytes were incubated for $30 \mathrm{~min}$ before injection of cRNA.

\section{Injection of cRNA into Oocytes}

Micropipettes were made using 8.9-cm glass tubes (Drummond Scientific Co., Broomall, PA) on a vertical puller (KOPF Needle and Pipette Puller; David Kopf Instruments, Tujunga, CA). Pipettes were trimmed and filled with sterile mineral oil using a modified syringe. Pipettes were fitted to the Drummond Nanoject automatic microinjector and were back-filled with cRNA (300 ng/ $/ \mu \mathrm{L}$, unless otherwise noted) or sterile water. Oocytes were transferred into a $60 \mathrm{~mm}$ Petri dish containing ND-96 media (96 $\mathrm{m} M \mathrm{NaCl}, 2 \mathrm{mM} \mathrm{KCl}, 1 \mathrm{~m} M$ $\mathrm{MgCl}_{2}, 1.8 \mathrm{mM} \mathrm{CaCl}_{2}$, and $5 \mathrm{~m} M$ HEPES, pH 7.5) for injection. Under a light microscope $(10 \times)$ individual mature oocytes (stage $\mathrm{V}$ and $\mathrm{VI}$ ) were injected in the vegetal pole or midline with approximately $46 \mathrm{~nL}$ of cRNA or water. Oocytes were returned to Barth's media and incubated at $18^{\circ} \mathrm{C}$ for $72 \mathrm{~h}$, unless otherwise specified in figure legends. Media was changed every 12 $\mathrm{h}$ during the incubation period and dead oocytes were removed when observed.

\section{2-Deoxy-D-Glucose Uptake by Oocytes}

Injected oocytes were washed once in antibiotic-free Barth's media and were randomly selected for treatment groups and transferred into a 24-well cell culture dish (Corning Inc., Corning, NY). 2-Deoxy-D- $\left[1-{ }^{3} \mathrm{H}\right]$-glucose $\left({ }^{3} \mathbf{H}-2-D G\right.$; Amersham Pharmacia Biotech Inc., Piscataway, NJ) was used as a radio-tracer for 2-DG (Sigma) uptake. Concentrations of nonradioactive 2-DG, ranging from 0 to $100 \mathrm{mM}$, were prepared in Barth's media containing no antibiotic. 2-Deoxy-D- $\left[1-{ }^{3} \mathrm{H}\right]$-glucose was added to each 2-DG solution at a concentration of 111 $\mathrm{kBq} / \mathrm{mL}$ (for kinetic assays) or $37 \mathrm{kBq} / \mathrm{mL}$ (for all other assays) of total volume. For assays conducted at one concentration, $5 \mathrm{mM}$ 2-DG was used. Hexose mixtures were stored at $4^{\circ} \mathrm{C}$ but were equilibrated to $22^{\circ} \mathrm{C}$ for $12 \mathrm{~h}$ before use. Three hundred microliters of each glucose analog solution $\left(2-\mathrm{DG}+{ }^{3} \mathrm{H}-2-\mathrm{DG}\right)$ was applied to each group of oocytes $(\mathrm{n}=5-25)$ for specified times, as indicated in figure legends. Times ranged from 0 to $90 \mathrm{~min}$; 0-min time points were conducted by applica- tion and immediate removal of 2-DG. When time was not a variable within an assay, oocytes were exposed to 2 -DG $+{ }^{3} \mathrm{H}-2-\mathrm{DG}$ for $15 \mathrm{~min}$. After application of 2-DG, the 24-well plate was gently agitated to ensure oocytes were suspended. Glucose uptake was halted by aspiration of the 2-DG solution and immediate application of approximately $1 \mathrm{~mL}$ of ice-cold PBS (Invitrogen Corp.) containing $0.1 \mathrm{~m} M$ phloretin (Sigma), an inhibitor of facilitative glucose transport (Krupka, 1985). The oocytes were washed 3 times within 1 min with PBS containing phloretin before suspension in $2 \mathrm{~mL}$ of the same solution, individually transferred to $7-\mathrm{mL}$ borosilicate glass scintillation vials (Fisher Scientific Co. LLC, Pittsburgh, PA) containing $0.5 \mathrm{~mL}$ of $1 \%$ (wt/vol) SDS and incubated for at least $1 \mathrm{~h}$. Five milliliters of Econo Safe liquid scintillation fluid (Atlantic Nuclear Inc., Rockland, MA) was added to the scintillation vial, which was then shaken until homogenous. Sample radioactivity was quantified using the Wallac 1400 DSA scintillation counter (PerkinElmer Wallac Inc., Gaithersburg, MD). Each sample was counted for $180 \mathrm{~s}$. Where indicated, the GLUT1-injected oocyte 2-DG uptake was corrected for the 2-DG uptake in water-injected oocytes exposed to the same 2-DG concentration and time as the GLUT1-injected oocytes.

\section{Inhibition of 2-DG Uptake by Cytochalasin B and Phloretin}

Oocytes injected with bGLUT1 cRNA and water were exposed to $5 \mathrm{mM} 2-\mathrm{DG}+{ }^{3} \mathrm{H}-2-\mathrm{DG}$ in the presence of various concentrations of cytochalasin B (CCB; Sigma) and phloretin (Sigma), 2 potent inhibitors of facilitative glucose transport (Sogin and Hinkle, 1980; Krupka, 1985). 2-Deoxy-D-glucose $+{ }^{3} \mathrm{H}-2-\mathrm{DG}$ with various concentrations of $\mathrm{CCB}$ and phloretin were applied to groups of 10 oocytes for $15 \mathrm{~min}$. Oocytes were then washed in ice-cold PBS, transferred into scintillation vials containing SDS, and counted as described above.

\section{Substrate Specificity: Hexose Sugar Inhibition Assays}

Inhibition of 2-DG uptake was measured in the presence of $30 \mathrm{~m} M$ concentrations of the following hexose sugars: L-glucose, D-glucose, D-mannose, D-fructose, 3-O-methylglucose, and D-galactose (Sigma). 2-DeoxyD-glucose was diluted to a final concentration of $5 \mathrm{mM}$ in Barth's media containing $30 \mathrm{~m} M$ individual inhibitor sugar. 2-Deoxy-D- $\left[1-{ }^{3} \mathrm{H}\right]$-glucose was added at a concentration of $37 \mathrm{kBq} / \mathrm{mL}$ of the final volume. Thirty millimolar L-glucose was used as a negative control for inhibition of 2-DG uptake (Gould et al., 1991). Three 
hundred microliters of 2-DG with ${ }^{3} \mathrm{H}-2$-DG and inhibitor hexose were applied to groups $(\mathrm{n}=20)$ of bGLUT1 cRNA- or water- injected oocytes. 2-Deoxy-D-glucose uptake from water-injected oocytes was subtracted from the cRNA-injected oocytes.

\section{Immunohistochemistry}

Oocytes were fixed for immunohistochemistry in $4 \%$ (wt/vol) paraformaldehyde for $3 \mathrm{~h}$ on ice. Oocytes were washed 3 times in PBS and snap frozen in optimal cutting temperature (OCT) compound (Sakura Finetek USA Inc., Torrance, CA). Oocyte blocks were sectioned and thaw-mounted on the surface of gelatincoated slides.

Tissue sections were equilibrated to $22^{\circ} \mathrm{C}$ before incubation in PBS containing 10\% (vol/vol) goat serum for $1 \mathrm{~h}$ at $22^{\circ} \mathrm{C}$ and then washed 2 times for 5 min with PBS. Sections were then exposed to $10 \mu \mathrm{g} / \mathrm{mL}$ of rabbit polyclonal anti-GLUT1 antibody (Millipore, Billerica, $\mathrm{MA}$ ) for $1 \mathrm{~h}$ at $22^{\circ} \mathrm{C}$ and washed 2 times for $5 \mathrm{~min}$ with PBS. Sections were incubated with Alexa Fluor 555-conjugated goat anti-rabbit IgG at a concentration of $2.5 \mu \mathrm{g} / \mathrm{mL}$ for $1 \mathrm{~h}$ in the dark at $22^{\circ} \mathrm{C}$. Sections were washed 2 times with PBS and once with ultrapure water before mounting under a cover slip with Aqua Poly/Mount (Polysciences Inc., Warrington, PA). Tissue sections were examined using the Zeiss LSM 510 META Confocal Laser Scanning Imaging System (Carl Zeiss Inc., Thornwood, NY) at the Microscopy Imaging Center, University of Vermont and visualized using the Zeiss LSM 5 image browser.

\section{Western Blot Analysis}

Total protein was isolated from cRNA- and waterinjected oocytes. Oocytes were washed in PBS and suspended in $10 \mu \mathrm{L}$ per oocyte of homogenization buffer (0.25 $\mathrm{m} M$ sucrose, $0.010 \mathrm{~m} M$ HEPES, $1 \mathrm{~m} M$ EGTA, and $2 \mathrm{mM} \mathrm{MgCl}{ }_{2}$ ) containing protease inhibitors (100 $\mathrm{m} M \mathrm{PMSF}, 1 \mathrm{mg} / \mathrm{mL}$ of pepstatin $\mathrm{A}$, and $1 \mathrm{mg} / \mathrm{mL}$ of leupeptin). Oocytes were disrupted by 10 strokes of a $200-\mu \mathrm{L}$ pipette tip. The homogenate was stored at $-20^{\circ} \mathrm{C}$ before use. Prior to use, samples were thawed on ice and centrifuged for $30 \mathrm{~s}$ to pellet yolk proteins. The Bradford assay (Bio-Rad Laboratories Inc., Hercules, $\mathrm{CA}$ ) was used to determine the protein concentration of the supernatant.

Oocyte cell homogenates were denatured at $100^{\circ} \mathrm{C}$ for $4 \mathrm{~min}$ in the presence of $10 \mu \mathrm{L}$ of $6 \times$ sample buffer [62.5 $\mathrm{m} M$ Tris (pH 6.8), $2 \%$ (wt/vol) SDS, $10 \%$ (vol/vol) glycerol, and $5 \%$ (vol/vol) 2- $\beta$-mercaptoethanol]. Proteins were electrophoresed on a $12 \%$ SDS-polyacrylamide gel and then electrophoretically transferred to a Hybond-P polyvinylidene difluoride (PVDF) membrane (Amersham Pharmacia Biotech Inc.) on ice. Membranes were then blocked in Tris-buffered saline [TBS; $20 \mathrm{mM}$ Tris (pH 7.4) and $137 \mathrm{mM} \mathrm{NaCl}$ containing 5\% (wt/vol) nonfat powdered milk (Bio-Rad Laboratories Inc.) and $0.01 \%$ (vol/vol) Tween-20 (Fisher Scientific Co. LLC). Membranes were washed 2 times in TBS containing $0.01 \%$ Tween for $5 \mathrm{~min}$ and then incubated for $1 \mathrm{~h}$ at $22^{\circ} \mathrm{C}$ with $1 \mu \mathrm{g} / \mathrm{mL}$ of rabbit anti-GLUT1 polyclonal antibody (Millipore) diluted in TBS-Tween containing $5 \%$ (wt/vol) nonfat milk. Membranes were washed 2 times in TBS-Tween and were then exposed to a horseradish peroxidase-conjugated anti-rabbit IgG secondary antibody (Amersham Pharmacia Biotech Inc.) at $22^{\circ} \mathrm{C}$ for $1 \mathrm{~h}$. Membranes were washed 3 times in TBS-Tween and once in TBS. The immune complex was detected using the West Pico Chemiluminescent Kit (Pierce Biotechnology Inc., Rockford, IL) and detected on x-ray film (Kodak, Rochester, NY). Bands were quantified by densitometry (Molecular Imager VersaDoc 4100 MP; Bio-Rad Laboratories Inc.).

\section{Statistical Analysis}

Data points in glucose uptake assays outside 1.5 times the inter-quartile range were designated as outliers and were disregarded for both water- and cRNAinjected oocytes. 2-Deoxy-D-glucose uptake in GLUT1 cRNA-injected oocytes was corrected by subtraction of the mean 2-DG uptake for water-injected oocytes at the corresponding concentration and incubation time; any resulting negative uptake values were disregarded. Statistical significance was determined by the TukeyKramer honestly significant difference (HSD) test, Welch ANOVA test, and Dunnett's one-way ANOVA, as indicated in individual figure legends, and the analyses were carried out using JMP 8.0 software (SAS Institute Inc., Cary, NC). Points indicate sample mean and error bars represent the standard error of the means. Plots and curve-fitting analysis for the Michaelis-Menten equation were carried out using GraphPad Prism 5.02 (GraphPad Software Inc., La Jolla, CA).

\section{RESULTS}

\section{Expression and Localization of Exogenous GLUT1 in Xenopus Oocytes}

To examine the transport kinetics of bGLUT1 in Xenopus oocytes, bGLUT1 cDNA was subcloned into the Xenopus specific expression vector, pSP64T (Gould et al., 1991). The open reading frame of bGLUT1 cDNA was inserted between the $5^{\prime}$ and $3^{\prime}$ untranslated flanking sequences of the Xenopus $\beta$-globin gene to form 
pSP64T-bGLUT1 and was in vitro transcribed. The hGLUT1 or bGLUT1 cRNA (15 ng/injection) or water were injected into oocytes; uninjected oocytes were also used to determine a baseline estimate of endogenous GLUT1 expression in Xenopus oocytes. The oocytes were harvested after $72 \mathrm{~h}$ of incubation and expression of GLUT1 protein was detected by Western blot analysis of whole oocyte lysates (Figure 1A), and quantified by densitometry (Figure 1B). Two distinct bands were detected in both the hGLUT1- and bGLUT1-injected oocytes at a molecular weight between 42 and $50 \mathrm{kDa}$ (data not shown), consistent with endogenous GLUT1 detected in the cells of a mouse mammary cell line HC11. Low levels of endogenous GLUT1 protein expression were detected in both the water-injected and uninjected oocytes, whereas injection of bGLUT1 or hGLUT1 cRNA injection increased the GLUT1 protein levels by 5 - to 10 -fold relative to the controls. The presence of 2 bands may be due to glycosylation of GLUT1 protein, a posttranslational modification that has been shown to be vital for transport activity by increasing the affinity of GLUT1 for glucose (Asano et al., 1991; Ahmed and Berridge, 1999).

Subcellular localization of hGLUT1, bGLUT1, and endogenous GLUT1 was assessed by immunofluorescence on cRNA- or water-injected oocytes (Figure 2). Bovine GLUT1 was primarily detected on the plasma membrane of the bGLUT1 cRNA-injected oocytes (Figure 2A). Human GLUT1 was localized to both the plasma membrane and the intracellular pool (Figure 2B). Water-injected oocytes had no detectable fluorescence staining (data not shown). The specificity of the secondary antibody was tested by exposure of bGLUT1 cRNA-injected oocytes to the secondary antibody alone or with normal rabbit serum (data not shown). These negative controls showed no detectable signal.

\section{Functional Characterization of Exogenous GLUT1 in Xenopus laevis Oocytes}

The functional transport activity of exogenous bGLUT1 in Xenopus oocytes was evaluated by exposing bGLUT1 cRNA-injected oocytes to $5 \mathrm{~m} M$ 2-DG containing ${ }^{3} \mathrm{H}-2-\mathrm{DG}$ for $15 \mathrm{~min}$. The water-injected and uninjected oocytes were used to determine the endogenous levels of glucose transport. 2-Deoxy-D-glucose uptake was significantly higher in the hGLUT1 and bGLUT1 cRNA-injected oocytes than in the water-injected and uninjected controls ( $\mathrm{n} \geq 88, P<0.0001$; Figure $3 \mathrm{~A}$ ). Oocytes injected with hGLUT1 and bGLUT1 cRNA had mean 2-DG uptakes of 580.0 and $568.7 \mathrm{pmol} /$ oocyte per $15 \mathrm{~min}$, respectively, whereas the water-injected oocytes had a mean 2-DG uptake of $44.6 \mathrm{pmol} /$ oocytes per $15 \mathrm{~min}$. No significant difference was observed in
A
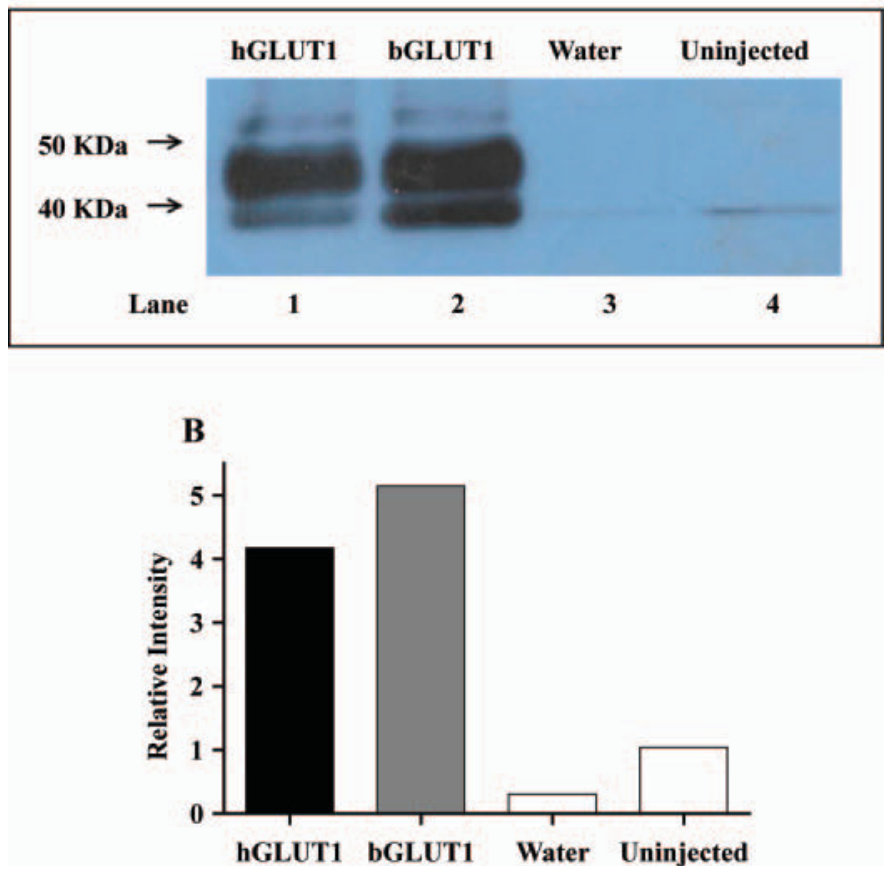

Figure 1. Expression of glucose transporter 1 (GLUT1) protein in Xenopus oocytes. Western blot analysis (A) was performed on homogenate of oocytes injected with either human GLUT1 (hGLUT1) or bovine GLUT1 (bGLUT1) cRNA. Water-injected and uninjected oocytes were used for detection of endogenous GLUT1 expression. Seventy-five micrograms of total protein was loaded in each lane. Relative GLUT1 band intensities were determined by densitometry (B). Color version available in the online PDF.

2-DG uptake among water-injected, uninjected oocytes, and uninjected oocytes treated with CCB.

To determine the quantity of cRNA needed to achieve maximal expression and transport activity of bGLUT1, we conducted 2-DG uptake assays in oocytes that were injected with various quantities of cRNA. No statistical difference in 2-DG uptake was observed in oocytes injected with 1.5 to $45 \mathrm{ng}$ of cRNA $(\mathrm{n}=10, P$ $=0.96$ ), in addition, no significant difference existed in 2-DG uptake between the 0.015- and 0.0015-ng cRNAinjected oocytes ( $\mathrm{n} \geq 6, P=0.16$; Figure 3B). However, significant difference was found between the $2 \mathrm{cRNA}$ groups of 1.5 to $45 \mathrm{ng}$ and 0.015 to $0.0015 \mathrm{ng}(P<$ 0.001). The concentration of $15 \mathrm{ng} /$ injection of cRNA was selected for use in further uptake assays to ensure maximal expression of bGLUT1 in the oocytes.

Facilitative GLUT are known to be inhibited by several pharmacological agents, including $\mathrm{CCB}$ and phloretin. To test whether, in our system, CCB and phloretin inhibited bGLUT1-mediated 2-DG transport, Xenopus oocytes injected with bGLUT1 cRNA were exposed to increasing concentrations of these 2 inhibitors during the 2-DG transport assay. The inhibitory 

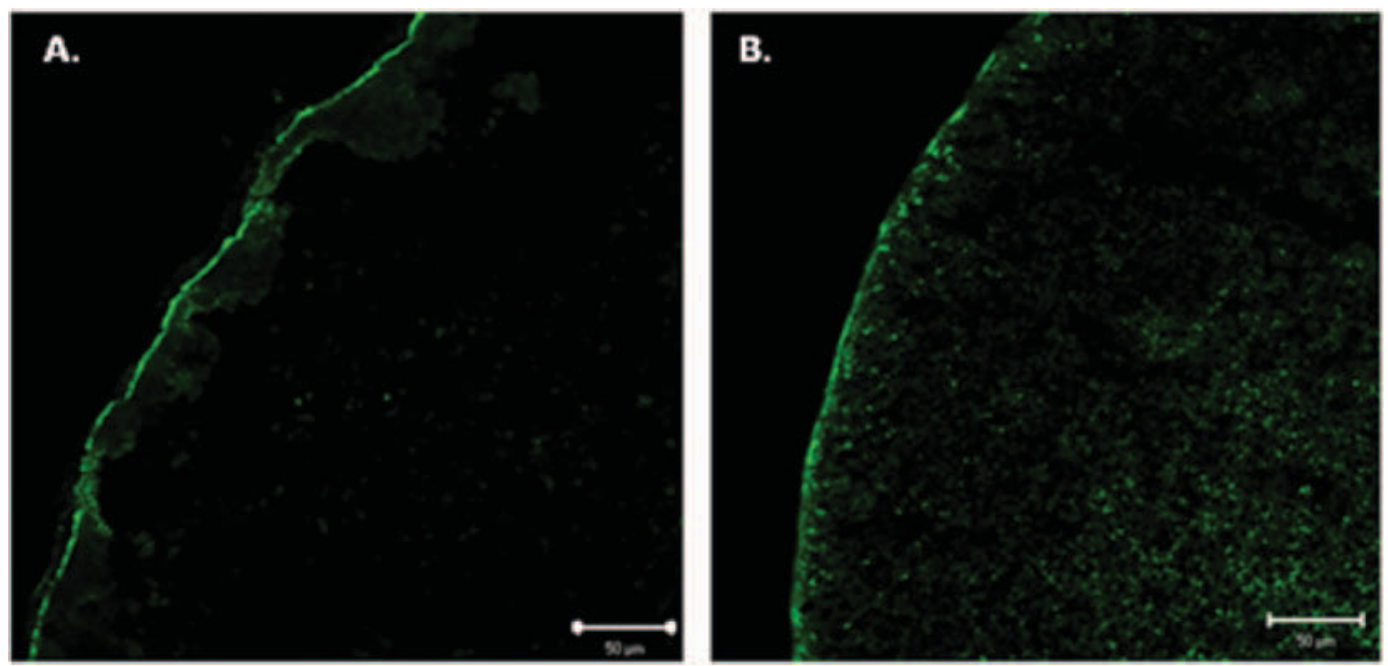

Figure 2. Immunofluorescence staining of exogenous glucose transporter 1 (GLUT1) in Xenopus oocytes injected with either bovine GLUT1 cRNA (A) or human GLUT1 cRNA (B). Scale bar $=50 \mu \mathrm{m}$. Color version available in the online PDF.

affect of CCB was tested by increasing concentrations from 0 to $100 \mu M$ (Figure 4A). Concentrations of CCB more than $25 \mu M$ significantly decreased bGLUT1mediated 2-DG uptake in oocytes (n $\geq 6, P<0.05$ ). 2-Deoxy-D-glucose uptake was highly reduced at 100 $\mu M$ CCB, from 1,165 to $47 \mathrm{pmol} /$ oocyte per $15 \min (P$ $<0.01$. Nonlinear one-phase decay analysis estimated an inhibitory plateau at $207.2 \mathrm{pmol}$ /oocyte per $15 \mathrm{~min}$ of 2-DG. The inhibitory effect of phloretin was tested by increasing concentrations from 0 to $500 \mu M$ (Figure 4B). Phloretin at concentrations $\geq 50 \mu M$ significantly decreased bGLUT1-mediated 2-DG uptake $(\mathrm{n} \geq 5, P<$ 0.001). Maximal inhibition of uptake was observed at concentrations $\geq 200 \mu M$. Nonlinear one-phase decay analysis estimated an inhibitory plateau at $718 \mathrm{pmol} /$ oocyte per 15 min.

The quaternary structure of GLUT is known to be regulated by intracellular ATP concentration (Heard et al., 2000). We, therefore, sought to determine if the lack of energy substrate in Barth's media affected 2-DG uptake by GLUT1. Oocytes injected with cRNA or water were cultured for $72 \mathrm{~h}$ in the presence or absence of 2.5 $\mathrm{m} M$ sodium pyruvate in Barth's media (Figure 5). The presence of sodium pyruvate had no effect on glucose uptake in oocytes injected with hGLUT1 (n $\geq 8, P=$ 0.65 ) or bGLUT1 (n $\geq 8, P=0.33)$ cRNA.

\section{Kinetic Analysis of bGLUT1 and hGLUT1 in Xenopus Oocytes}

Determination of transport $K_{m}$ and $V_{\max }$ values using Michaelis-Menten enzyme kinetics were carried out using 15-min incubations, which were found to be within the linear range of uptake for bGLUT1 (data not shown), consistent with previous studies of hGLUT1 (Gould et al., 1991). Kinetic analysis of bGLUT1 and hGLUT1 was conducted using various concentrations of 2-DG, ranging from 0 to $50 \mathrm{~m} M$. The analysis was repeated on oocytes from multiple frogs (9 assays for bGLUT1 and 5 for hGLUT1). A representative assay for each bGLUT1 and hGLUT1 are shown in Figure 6 . Michaelis-Menten curve fit analysis of 2-DG uptake in GLUT1-injected oocytes after correcting for the uptake into the water-injected oocytes revealed $K_{m}$ values ranging from 5.1 to $14.0 \mathrm{~m} M$ for bGLUT1 and from 7.9 to $17.8 \mathrm{~m} M$ for hGLUT1. The mean $K_{m}$ for bGLUT1 and hGLUT1 were $9.8 \pm 3.0$ and $11.7 \pm 3.6 \mathrm{mM}$, respectively. No statistical difference was observed between the $K_{m}$ of bGLUT1 and hGLUT1 $(P=0.24)$. The $V_{\max }$ values ranged from 2,582 to $4,914 \mathrm{pmol} /$ oocyte per 15 min for bGLUT1 and 2,773 to 4,914 pmol/oocyte per $15 \mathrm{~min}$ for hGLUT1. The mean $V_{\max }$ was $3,567 \pm 721$ pmol/oocyte per $15 \mathrm{~min}$ for bGLUT1 and 3,947 \pm 790 pmol/oocyte per 15 min for hGLUT1.

\section{Determination of bGLUT1 Substrate Specificities}

The substrate specificity of bGLUT1 was determined by the competitive inhibition of hexose sugars $(30 \mathrm{mM})$ on 2-DG uptake in oocytes expressing bGLUT1 (Figure 7). L-Glucose was used as a negative control for the inhibition as GLUT have stereoselectivity for the D-enantiomer (Keller et al., 1989). The uptake in the presence of L-glucose was consistent with 2-DG uptake in noninhibitory conditions. The positive controls, 2-DG and D-glucose, both significantly inhibited the uptake to $480 \pm 20 \mathrm{pmol} /$ oocyte per $15 \min (\mathrm{n} \geq 18$, $P<0.0001)$. 3-O-methylglucose, a nonmetabolizable 
glucose analog, significantly inhibited 2-DG uptake (n $\geq 17, \quad P<0.01$ ). 2-Deoxy-D-glucose uptake was also inhibited by D-mannose and D-galactose $(\mathrm{n} \geq 14, P<$ $0.01)$, but not by D-fructose $(\mathrm{n}=19, P=0.18)$.

\section{DISCUSSION}

\section{Characterization of bGLUT1 Expression and 2-DG Transport in Xenopus Oocytes}

The objective of our study was to establish the Xenopus oocyte as a model for the expression of bGLUT1 and determine the functional kinetic properties of the transporter in this model. The Xenopus oocyte is an effective model for the study of GLUT, as oocytes have low levels of endogenous glucose transport activity and allow for the expression of exogenous GLUT (Keller and Mueckler, 1990). In Xenopus oocytes, glucose transport is not limited by phosphorylation of the sugar by hexokinase; therefore, the model can accurately simulate the steep concentration gradient across the basolateral membrane within the mammary gland (Gould et al., 1991). Our results demonstrated that bGLUT1 can be successfully expressed in Xenopus oocytes and that the protein localizes to the plasma membrane. We have shown that bGLUT1 is functionally active in oocytes and that bGLUT1-mediated transport can be inhibited by $\mathrm{CCB}$ and phloretin, two widely used inhibitors of facilitative GLUT.

In our study, water-injected and uninjected oocytes displayed only very low levels of endogenous GLUT1 expression and very low, but detectable levels of 2-DG uptake, a finding consistent with previous reports (Keller and Mueckler, 1990). This uptake may be mediated by low endogenous GLUT1 present in frog embryos (Suzawa et al., 2007). We observed, however, no inhibitory effect of CCB on 2-DG uptake in uninjected oocytes. Thus, it appears that the 2-DG uptake in water-injected oocytes may be attributable to radioactivity retained on the oocytes after washing, and is not solely a function of endogenous GLUT-mediated transport.

The 2-DG uptake in oocytes injected with bGLUT1 cRNA was not affected by adding sodium pyruvate, an energy substrate for the oocytes (Liu, 2006). This indicates that, in our culture conditions, a lack of energy in the growth media did not affect the function or expression of exogenous bGLUT1 protein in oocytes. Another potential rate-limiting step to bGLUT1 production in oocytes was the amount of bGLUT1 cRNA injected. Our results indicated that $1.5 \mathrm{ng}$ of cRNA is enough to saturate the translational or trafficking machinery of the oocytes, as we observed no further increase in 2-DG
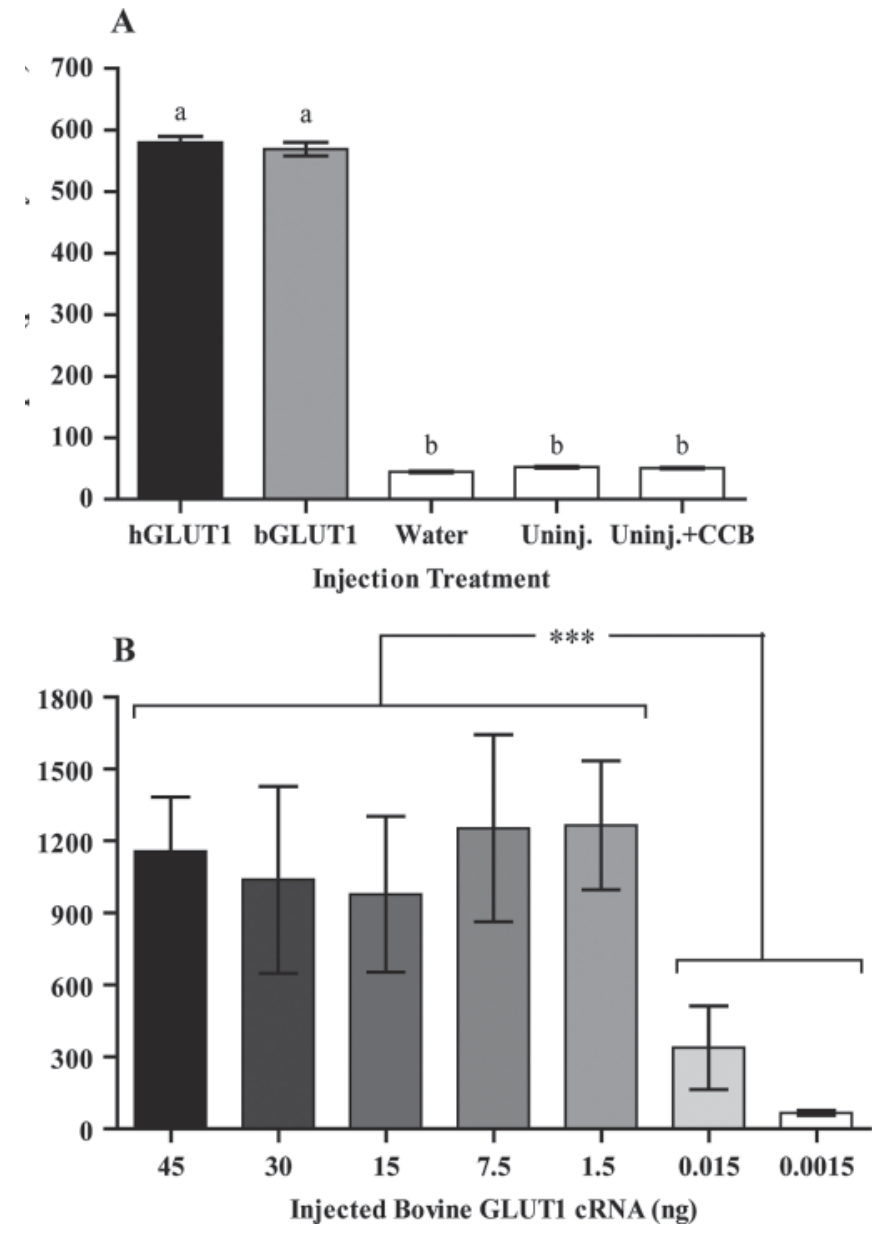

Figure 3. Transport activity of exogenous glucose transporter 1 (GLUT1) in Xenopus oocytes. A: Xenopus oocytes were injected with human GLUT1 (hGLUT1) or bovine GLUT1 (bGLUT1) cRNA (15 ng), water, or were not injected (Uninj.). One hundred micromolar cytochalasin B (CCB) was added to the uptake reaction of a group of uninjected oocytes (Uninj.+CCB). Oocytes were exposed to $5 \mathrm{mM}$ 2-deoxy-D-glucose (2-DG) containing $37 \mathrm{kBq} / \mathrm{mL}$ of 2-deoxy-D-[1- $\left.{ }^{3} \mathrm{H}\right]$ glucose $\left({ }^{3} \mathrm{H}-2-\mathrm{DG}\right)$ for $15 \mathrm{~min}$. Means of 2-DG uptake in the cRNA-injected oocytes versus water-injected or uninjected controls were compared using the Tukey-Kramer honestly significant difference (HSD) test; error bars represent standard errors of the means $(P<0.001)$. Different letters ( $\mathrm{a}$ and $\mathrm{b}$ ) indicate differences at $P<0.05$. B: Oocytes were injected with 0.0015 to $45 \mathrm{ng}$ of bovine GLUT1 cRNA and subjected to the transport analysis as in A. Data represent the uptake in the cRNA-injected oocytes after subtracting the uptake in waterinjected oocytes. Statistical significance was determined by Welch ANOVA; ${ }^{* * *} P<0.001$, comparing groups as indicated in the figure.

uptake in oocytes injected with higher concentrations of bGLUT1 cRNA.

\section{Kinetic Analysis of bGLUT1 in Xenopus Oocytes}

The kinetic parameters of bGLUT1 were determined in Xenopus oocytes under zero-trans conditions. The linear range of uptake for bGLUT1 was found to be 


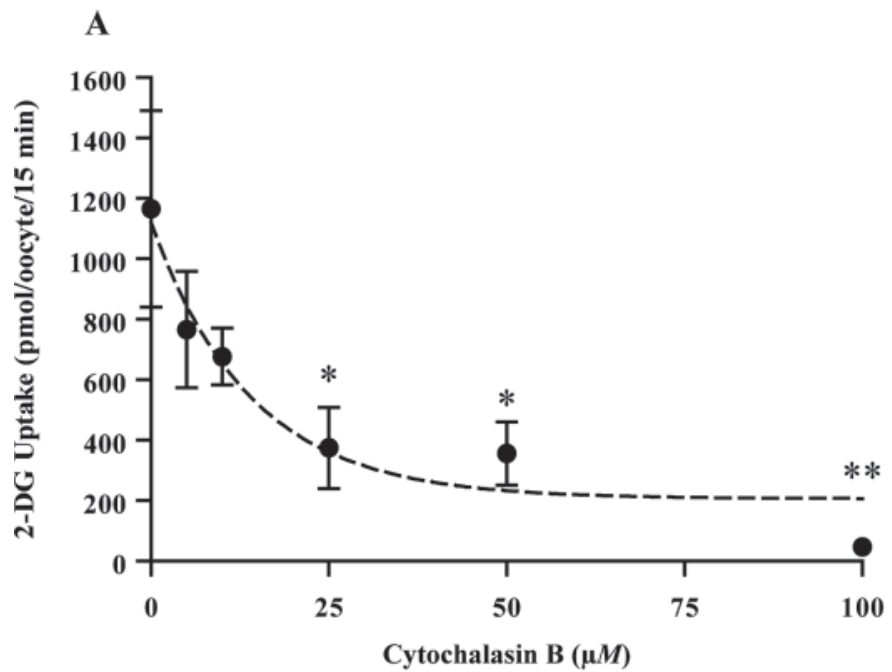

B

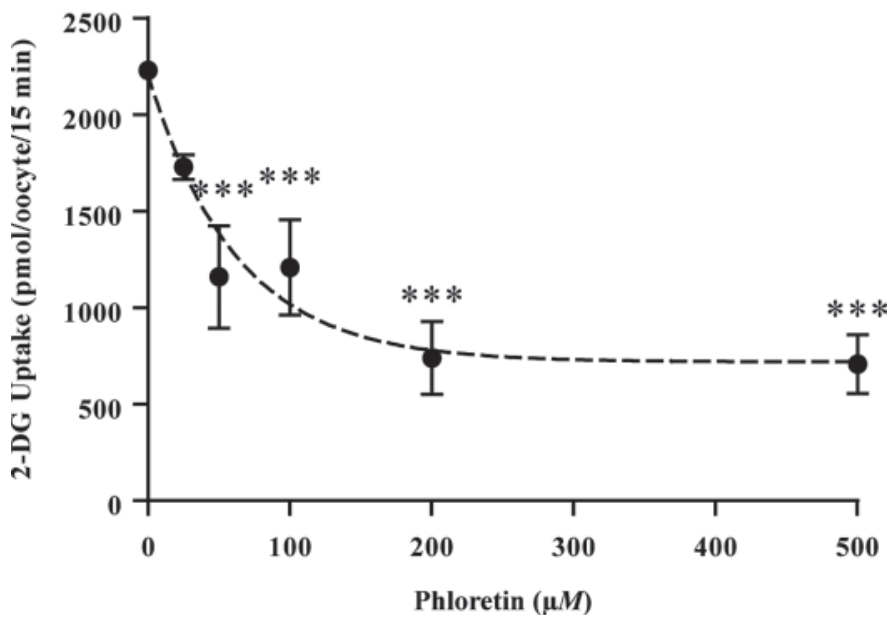

Figure 4. Inhibition of bovine glucose transporter 1 (GLUT1)mediated 2-deoxy-D-glucose uptake by cytochalasin B and phloretin in Xenopus oocytes. Oocytes were injected with bovine GLUT1 cRNA or water. Oocyte 2-deoxy-D-glucose (2-DG) uptake was measured after exposure of oocytes to $5 \mathrm{mM} 2$-DG and $37 \mathrm{kBq} / \mathrm{mL}$ of 2-deoxyD- $\left[1-{ }^{3} \mathrm{H}\right]$-glucose $\left({ }^{3} \mathrm{H}-2-\mathrm{DG}\right)$ for $15 \mathrm{~min}$ and various concentrations of cytochalasin B (A) or phloretin (B). 2-Deoxy-D-glucose uptake from water-injected oocytes was subtracted from cRNA-injected oocytes. A one-phase nonlinear decay model was used to identify the inhibition trend. Statistical analysis was conducted using Dunnett's ANOVA, with $0 \mu M$ cytochalasin B or phloretin as the control group. Statistical significance was observed at $P<0.05\left(^{*}\right), P<0.01\left(^{* *}\right)$, and $P<$ $0.001(* * *)$.

within 45 min of incubation with the substrate, which is consistent with previous studies of hGLUT1 (Gould et al., 1991). In our kinetic assay with $15 \mathrm{~min}$ of exposure to substrate, bGLUT1 exhibited MichaelisMenten type transport kinetics and was saturated at 2-DG concentrations greater than $25 \mathrm{mM}$. Bovine GLUT1 has a mean $K_{m}$ of $9.8 \pm 3.0 \mathrm{~m} M$, which is not statistically different from the $K_{m}$ of hGLUT1 (11.7

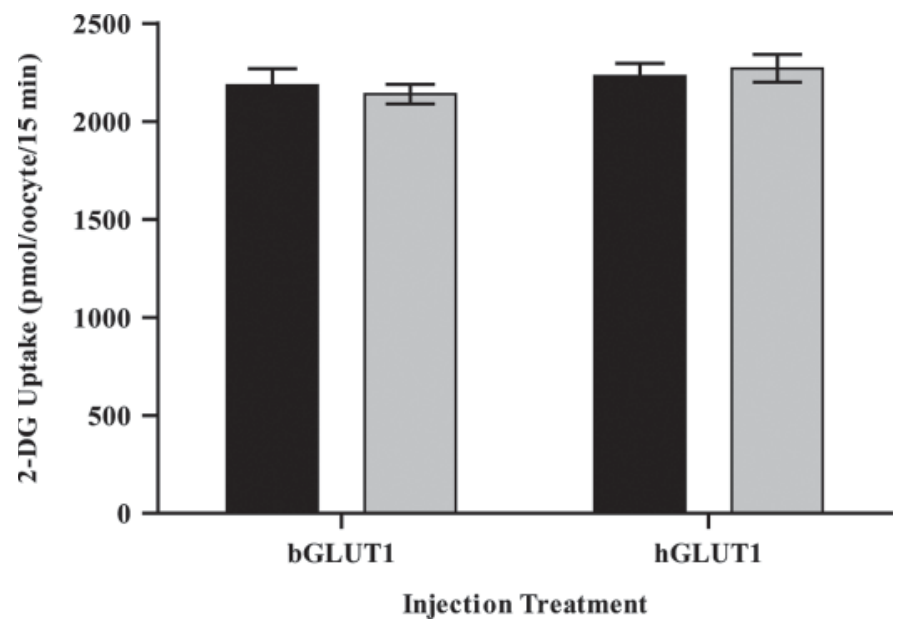

Figure 5. Effect of sodium pyruvate as an energy source on 2deoxy-D-glucose (2-DG) uptake in Xenopus oocytes. Oocytes were injected with bovine or human glucose transporter 1 (GLUT1) cRNA or water. All oocytes were incubated for $72 \mathrm{~h}$ in the presence (black bars) or absence (gray bars) of $2.5 \mathrm{mM}$ sodium pyruvate in Barth's media. Oocytes were exposed to $5 \mathrm{mM} 2-\mathrm{DG}$ and $37 \mathrm{kBq} / \mathrm{mL}$ of 2-deoxyD- $\left[1-{ }^{3} \mathrm{H}\right]$-glucose $\left({ }^{3} \mathrm{H}-2-\mathrm{DG}\right)$ for $15 \mathrm{~min}$. 2-Deoxy-D-glucose uptake in water-injected oocytes was subtracted from cRNA-injected oocytes. Statistical analysis was conducted using the Tukey-Kramer honestly significant difference (HSD) test; error bars represent standard errors of the means.

$\pm 3.6 \mathrm{mM})$. These $K_{m}$ values are between the previously reported $K_{m}$ for rat GLUT1 (6.9 m $M$; Burant and Bell, 1992) and hGLUT1 (17 mM; Gould et al., 1991). These differences may be due to different experimental conditions. Nevertheless, our study does not support our hypothesis that bGLUT1 transports glucose with a higher affinity than the hGLUT1. Sequence alignment of bGLUT1 and hGLUT1 shows only 13 amino acid differences (Zhao and Keating, 2007b). These amino acids may not play a critical role in glucose transport activity of the GLUT1.

The $K_{m}$ of a plasma membrane transporter higher than the concentration of substrate in the blood would generally indicate that the transporter is functioning within its linear range of transport. This allows the transporter to decrease or increase its rate of uptake based on fluctuations in its substrate concentration in the blood. If the transport property of bGLUT1 in bovine cells in vivo is similar to that in Xenopus oocytes, our data indicate that in vivo, bGLUT1 on the plasma membrane is approximately $30 \%$ saturated at the blood glucose level of 3 to $3.5 \mathrm{~m} M$ (Faulkner and Peaker, 1987) and glucose uptake by the cells would increase when blood glucose concentration rises without increase in GLUT1 protein abundance. Supporting this notion is that the $K_{m}$ value of glucose uptake has been shown to be $8.29 \mathrm{mM}$ in the mammary epithelial cells in which GLUT1 is the predominant GLUT (Xiao and Cant, 


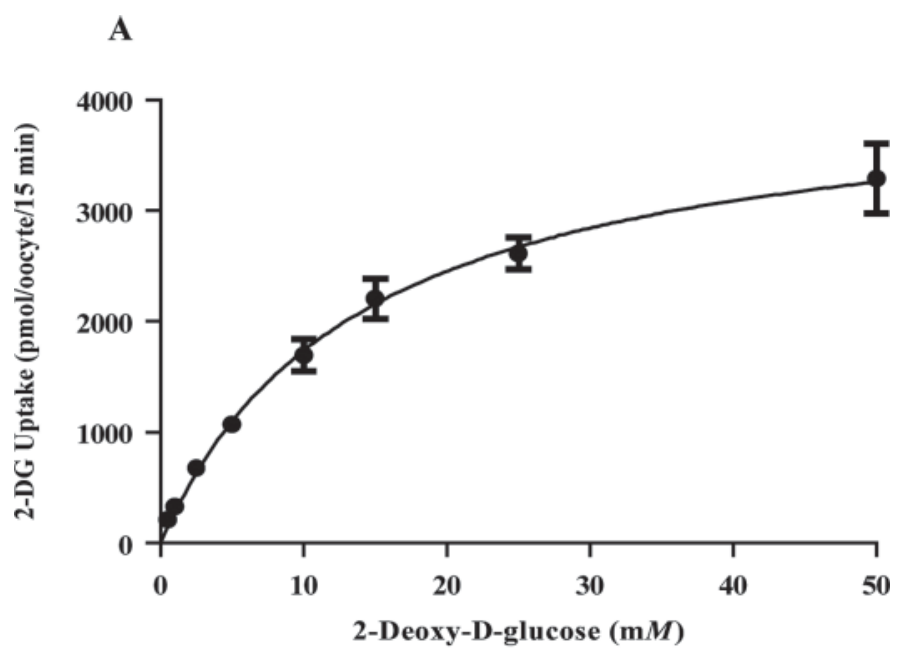

B

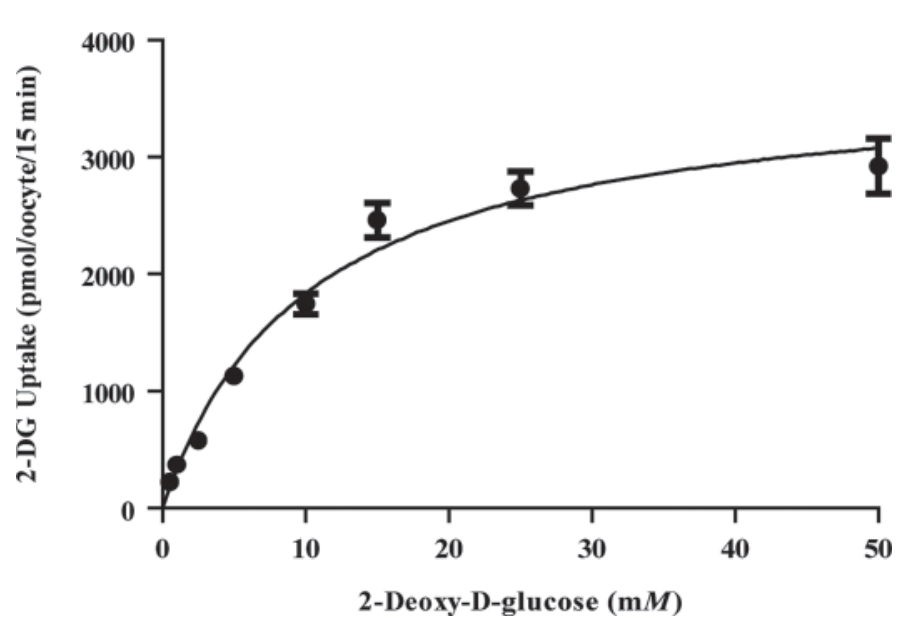

Figure 6. Kinetic analysis of 2-deoxy-D-glucose (2-DG) uptake by bovine (A) and human glucose transporter 1 (GLUT1; B) in Xenopus oocytes. Oocytes were injected with bovine or human GLUT1 cRNA or water and exposed to various 2-DG concentrations containing 111 $\mathrm{kBq} / \mathrm{mL}$ of 2-deoxy-D-[1- $\left.{ }^{3} \mathrm{H}\right]$-glucose $\left({ }^{3} \mathrm{H}-2-\mathrm{DG}\right)$ for $15 \mathrm{~min}$. Points represent 2-DG uptake in GLUT1-injected oocytes after correction for the uptake into water-injected oocytes. One representative assay is shown for each bGLUT1 and hGLUT1. Michaelis-Menten nonlinear analysis was conducted in GraphPad Prism 5 (GraphPad Software Inc., La Jolla, CA). Error bars represent standard errors of the means.

2003). Increasing blood glucose levels by intravenous or intestinal glucose infusion enhances glucose uptake by the mammary gland and milk production in dairy cows (Hurtaud et al., 2000; Huhtanen et al., 2002; Rigout et al., 2002).

\section{Substrate Specificity of bGLUT1}

Substrate specificity of bGLUT1 was assessed by inhibition of 2-DG uptake by different hexose sugars, including galactose, mannose, and fructose. The inhibitory effects of hexose sugars were compared with that

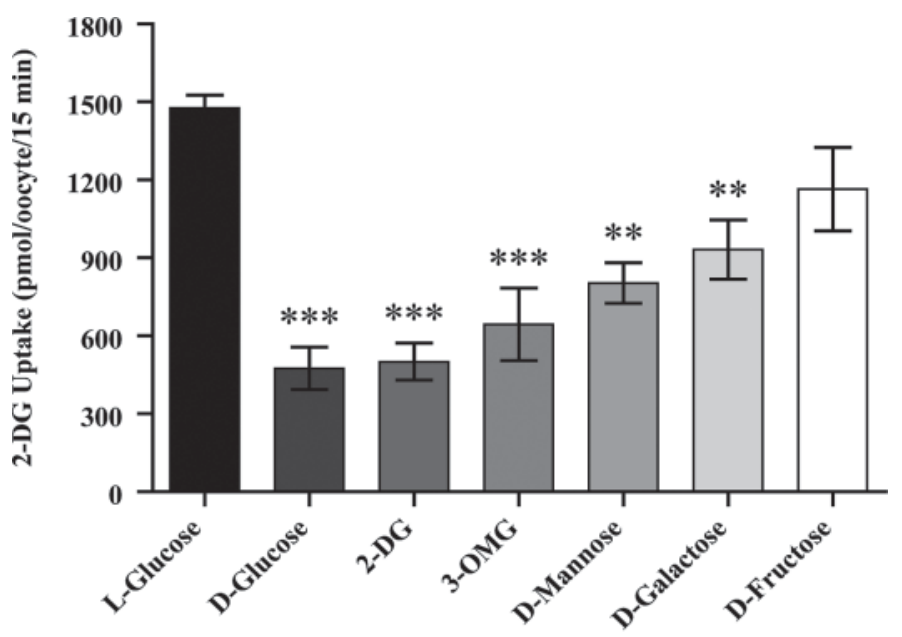

Hexose Inhibitor

Figure 7. Inhibition of bovine glucose transporter 1 (GLUT1)mediated uptake of 2-deoxy-D-glucose (2-DG) by hexose substrates in Xenopus oocytes. Oocytes were injected with bovine GLUT1 cRNA or water and were exposed to $30 \mathrm{~m} M$ concentrations of each inhibitor sugar, $5 \mathrm{mM} 2$-DG, and $37 \mathrm{kBq} / \mathrm{mL}$ of 2 -deoxy-D- $\left[1-{ }^{3} \mathrm{H}\right]$-glucose $\left({ }^{3} \mathrm{H}-2-\right.$ DG) for $15 \mathrm{~min}$. 2-Deoxy-D-glucose uptake from water-injected oocytes was subtracted from cRNA-injected oocytes. Statistical analysis was conducted using Dunnett's ANOVA, with L-glucose as the control group. Statistical significance was observed at $P<0.01\left(\right.$ ** $\left.^{*}\right)$ and $P<$ 0.001 (***); error bars represent standard errors of the means. 3-OMG $=3$-O-methylglucose.

of L-glucose, which has been previously shown to not be transported by GLUT1 (Gould et al., 1991). We observed that D-mannose and D-galactose inhibit 2-DG transport, whereas D-fructose does not. The inhibitory effect of D-glucose was similar to that of 2-DG, indicating that in Xenopus, oocyte bGLUT1 transports the glucose analog 2-DG as efficiently as D-glucose. The blood levels of hexose sugars other than glucose are generally low; thus, glucose is the major substrate for GLUT1 on the plasma membrane. In mammary epithelial cells, however, both glucose and galactose are transported into the Golgi apparatus for lactose synthesis. Glucose transporter 1 has been shown to be present in Golgi membrane of the mammary epithelial cells (Nemeth et al., 2000) and, therefore, bGLUT1 may be involved in the transport of both glucose and galactose into the Golgi apparatus.

\section{CONCLUSIONS}

Glucose transporter 1 plays a major role in the uptake of glucose necessary for milk production in the mammary gland. Here, we have characterized bGLUT1 transport kinetics and substrate specificities in Xenopus oocytes. Our study provides insight into the function of GLUT1 in glucose uptake in the mammary gland. However, it is important to point out that GLUT1 may 
function differently in Xenopus oocyte than in vivo. It may be necessary to study the transport kinetics of GLUT1 in mammary epithelial cells in future studies to obtain more accurate transport properties of GLUT1 in situ. The bovine mammary gland also expresses other isoforms of GLUT, including GLUT8 and 12 (Zhao et al., 2004; Miller et al., 2005). The transport kinetics and properties of these transporters should also be studied to fully understand the glucose uptake process in the mammary gland.

\section{ACKNOWLEDGMENTS}

We acknowledge Gwyn Gould (University of Glasgow, Glasgow, UK) for providing us the hGLUT1 construct and pSP64T vector as well as extensive technical advice. Thanks to Mike Mueckler (Washington University, St. Louis, MO) and Thomas McFadden, Chris Cheeseman, and Katarzyna Witkowska (all of University of Alberta, Edmonton, AB, Canada) for their technical advice. We also thank Sheryl White (University of Vermont, Burlington) for making the bGLUT1 construct, Marilyn Wadsworth (University of Vermont) for assistance with confocal microscopy, and Alan Howard (University of Vermont) for statistical support. This project was supported by National Research Initiative Competitive Grant no. 2007-35206-18037 from the USDA National Institute of Food and Agriculture, Washington, DC (to F.-Q. Zhao).

\section{REFERENCES}

Ahmed, N., and M. V. Berridge. 1999. N-glycosylation of glucose transporter-1 (Glut-1) is associated with increased transporter affinity for glucose in human leukemic cells. Leuk. Res. 23:395-401.

Asano, T., H. Katagiri, K. Takata, J.-L. Lin, H. Ishihara, K. Inukai, K. Tsukuda, M. Kikuchi, H. Hirano, Y. Yazaki, and Y. Oka. 1991. The role of $N$-glycosylation of GLUT1 for glucose transport activity. J. Biol. Chem. 266:24632-24636.

Burant, C. F., and G. I. Bell. 1992. Mammalian facilitative glucose transporters: Evidence for similar substrate recognition sites in functionally monomeric proteins. Biochemistry 31:10414-10420.

Colville, C. A., M. J. Seatter, T. J. Jess, G. W. Gould, and H. M. Thomas. 1993. Kinetic analysis of the liver-type (GLUT2) and brain-type (GLUT3) glucose transporters in Xenopus oocytes: Substrate specificities and effects of transport inhibitors. Biochem. J. 290:701-706.

Corpe, C. P., F. J. Bovelander, C. M. Munoz, J. H. Hoekstra, I. A. Simpson, O. Kwon, M. Levine, and C. F. Burant. 2002. Cloning and functional characterization of the mouse fructose transporter, GLUT5. Biochim. Biophys. Acta 1576:191-197.

Faulkner, A., and M. Peaker. 1987. Regulation of mammary glucose metabolism in lactation. Pages 535-562 in The Mammary Gland, Development, Regulation and Function. M. C. Neville and C. W. Daniel, ed. Plenum Press, New York, NY.

Finucane, K. A., T. B. McFadden, J. P. Bond, J. J. Kennelly, and F. Q. Zhao. 2008. Onset of lactation in the bovine mammary gland: Gene expression profiling indicates a strong inhibition of gene expression in cell proliferation. Funct. Integr. Genomics 8:251-264.

Gould, G. W., H. M. Thomas, T. J. Jess, and G. I. Bell. 1991. Expression of human glucose transporters in Xenopus oocytes: Kinetic characterization and substrate specificities of the erythrocyte, liver, and brain isoforms. Biochemistry 30:5139-5145.

Heard, K. S., N. Fidyk, and A. Carruthers. 2000. ATP-dependent substrate occlusion by the human erythrocyte sugar transporter. Biochemistry 39:3005-3014.

Huhtanen, P., A. Vanhatalo, and T. Varvikko. 2002. Effects of abomasal infusions of histidine, glucose, and leucine on milk production and plasma metabolites of dairy cows fed grass silage diets. J. Dairy Sci. 85:204-216.

Hurtaud, C., S. Lemosquet, and H. Rulquin. 2000. Effect of graded duodenal infusions of glucose on yield and composition of milk from dairy cows. 2. Diets based on grass silage. J. Dairy Sci. 83:2952-2962.

Keller, K., and M. Mueckler. 1990. Different mammalian facilitative glucose transporters expressed in Xenopus oocytes. Biomed. Biochim. Acta 49:1201-1203.

Keller, K., M. Strube, and M. Mueckler. 1989. Functional expression of the human HepG2 and rat adipocyte glucose transporters in Xenopus oocytes. Comparison of kinetic parameters. J. Biol. Chem. 264:18884-18889.

Krupka, R. M. 1985. Asymmetrical binding of phloretin to the glucose transport system of human erythrocytes. J. Membr. Biol. $83: 71-80$.

Liu, X. J. 2006. Xenopus Protocols: Cell biology and Signal Transduction. Humana Press, Totowa, NJ

Miller, P. J., K. A. Finucane, M. Hughes, and F. Q. Zhao. 2005. Cloning and expression of bovine glucose transporter GLUT12. Mamm. Genome 16:873-883.

Mueckler, M., C. Caruso, S. A. Baldwin, M. Panico, I. Blench, H. R. Morris, W. J. Allard, G. E. Lienhard, and H. F. Lodish. 1985. Sequence and structure of a human glucose transporter. Science 229:941-945.

Nemeth, B. A., S. W. Tsang, R. S. Geske, and P. M. Haney. 2000. Golgi targeting of the GLUT1 glucose transporter in lactating mouse mammary gland. Pediatr. Res. 47:444-450.

Nishimura, H., F. V. Pallardo, G. A. Seidner, S. Vannucci, I. A. Simpson, and M. J. Birnbaum. 1993. Kinetics of GLUT1 and GLUT4 glucose transporters expressed in Xenopus oocytes. J. Biol. Chem. 268:8514-8520.

Peaker, M. 1977. Mechanism of milk secretion: Milk composition in relation to potential difference across the mammary epithelium. J. Physiol. 270:489-505.

Rigout, S., S. Lemosquet, J. E. van Eys, J. W. Blum, and H. Rulquin. 2002. Duodenal glucose increases glucose fluxes and lactose synthesis in grass silage-fed dairy cows. J. Dairy Sci. 85:595-606.

Sogin, D. C., and P. C. Hinkle. 1980. Binding of cytochalasin B to human erythrocyte glucose transporter. Biochemistry 19:5417-5420.

Suzawa, K., A. Yukita, T. Hayata, T. Goto, H. Danno, T. Michiue, K. W. Cho, and M. Asashima. 2007. Xenopus glucose transporter 1 (xGLUT1) is required for gastrulation movement in Xenopus laevis. Int. J. Dev. Biol. 51:183-190.

Wright, E. M., and E. Turk. 2004. The sodium/glucose cotransport family SLC5. Pflugers Arch. 447:510-518.

Xiao, C., and J. P. Cant. 2003. Glucose transporter in bovine mammary epithelial cells is an asymmetric carrier that exhibits cooperativity and trans-stimulation. Am. J. Physiol. Cell Physiol. 285:C1226-C1234.

Zhao, F.-Q., D. R. Glimm, and J. J. Kennelly. 1993. Distribution of mammalian facilitative glucose transporter messenger RNA in bovine tissues. Int. J. Biochem. 25:1897-1903.

Zhao, F.-Q., and A. F. Keating. 2007a. Expression and regulation of glucose transporters in the bovine mammary gland. J. Dairy Sci. 90(Suppl 1.):E76-E86.

Zhao, F.-Q., and A. F. Keating. 2007b. Functional properties and genomics of glucose transporters. Curr. Genomics 8:113-128.

Zhao, F.-Q., P. J. Miller, E. H. Wall, Y.-C. Zheng, B. Dong, M. C. Neville, and T. B. McFadden. 2004. Bovine glucose transporter GLUT8: Cloning, expression, and developmental regulation in mammary gland. Biochim. Biophys. Acta 1680:103-113. 